Pacific Journal of Mathematic 


\title{
INVOLUTIONS OF THE 3-SPHERE WHICH FIX 2-SPHERES
}

\section{ROBERT CRAGGS}

\begin{abstract}
We show in that the space of involutions of the 3-sphere whose fixed point sets are 2-spheres is pathwise and locally pathwise connected. From Smith theory it is known that these involutions are orientation reversing. The fixed point sets need not be tame 2-spheres; Bing and others have many examples of involutions of the 3 -sphere whose fixed point sets are wild 2-spheres.
\end{abstract}

In order to prove the connectivity theorem ( $\S 6)$ just mentioned we develop an approximation theory for involutions of the 3-sphere in $\S$ 's 3,4 . Some of the results there are interesting in their own right. Corollary 3.1 states that involutions which fix wild 2-spheres can be approximated by involutions which fix tame 2-spheres. Theorem 4.6 states that if an involution $g$ fixing a 2-sphere $R$ approximates an involution $f$ fixing a 2-sphere $S$ very closely then $R$ approximates $S$. We also make use of Theorem 5.2, a modified version of the Alexander deformation theorem, which states that if the boundary of a 3-cell $C$ in the 3-sphere approximates a given 2-sphere very closely then very small homeomorphisms of $C$ onto itself which fix $\mathrm{Bd}(C)$ can be deformed back to the identity by small isotopies of $C$ which fix $\mathrm{Bd}(C)$.

Notation. Most of our notation conventions are discussed in [12]. We mention a few items here.

With one exception which we note later in this paragraph $\rho$ denotes the metric on a metric space. In the case of Euclidean space $E^{n}$ and its subspaces $\rho$ is given by $\rho(x, y)=\left\{\Sigma\left(x_{i}-y_{i}\right)^{2}\right\}^{1 / 2}$ where $x=\left(x_{1}, \cdots, x_{n}\right)$, $y=\left(y_{1}, \cdots, y_{n}\right)$. For spaces $X, Y, \mathscr{H}(X, Y)$ denotes the space of homeomorphisms of $X$ into $Y$ with the compact open topology. If $X$ is compact and $Y$ metric $\mathscr{H}(X, Y)$ is a metric space with metric $d$ given by $d(f, g)=\sup \{\rho(f(x), g(x)) \mid x \in X\}$.

An isotopy $H_{t}(a \leqq t \leqq b)$ of a space $X$ into itself is a continuous, one parameter family of homeomorphisms of $X$ into itself or alternately a parameterized path in $\mathscr{H}(X, X)$. In case $X$ is a metric space we say $H_{t}$ is an $\varepsilon$-isotopy provided the track under $H_{t}$ of each point $x$ of $X-\left\{H_{t}(x) \mid t \in[a, b]\right\}$ - has diameter less than $\varepsilon$. An $\varepsilon$-set in a metric space $X$ is a subset of $X$ of diameter less than $\varepsilon$. If $x \in X$ then $N(X, x, \varepsilon)$ denotes $\{y \in X \mid \rho(x, y)<\varepsilon\}$. If $Y \subseteq X$ then $N(X, Y, \varepsilon)$ denotes $\bigcup\{N(X, y, \varepsilon) \mid y \in Y\}$.

We denote the 3 -sphere, the unit sphere in Euclidean 4-space $E^{4}$, by $\Sigma$. We denote by $\mathscr{F}$ the subspace of $\mathscr{H}(\Sigma, \Sigma)$ consisting of those 
involutions of $\Sigma$ whose fixed point sets are 2-spheres and by $\mathscr{G}$ the subspace of $\mathscr{F}$ consisting of those involutions whose fixed point sets are tame 2-spheres. If $S$ is a 2 -sphere in $\Sigma, \mathscr{F}(S), \mathscr{G}(S)$ denote the subspaces of $\mathscr{F}, \mathscr{G}$ consisting of elements which have $S$ for a fixed point set. A crumpled cube is a space homeomorphic to the closure of the bounded component of the complement of a 2-sphere in $E^{3}$.

We assume the reader is familiar with the works of Moise and Bing on the triangulation theorem and Hauptvermutung for 3-manifolds $[4,6,17,18,19]$ as well as some of the elementary consequences of these works-for example from [17, Corollary to Theorem 1] that tame 2 -spheres bound pairs of 3-cells in $\Sigma$ and that disjoint tame 2-spheres in $\Sigma$ cobound annuli.

The following theorem which combines special cases of $[12, \S 9]$ and $[13, \S 8]$ will be applied in several places in this paper:

Theorem 1.1. Suppose $M$ is a (pwl) 3-manifold without boundary, $K$ is a compact polyhedron with no local cut points, $f$ is a homeomorphism of $K$ into $M$, and $\varepsilon>0$.

There is a $\delta>0$ such that:

(a) if $f_{0}$ and $f_{1}$ are (pwl) homeomorphisms of $K$ onto tame sets in $M$ with $d\left(f, f_{e}\right)<\delta(e=0,1)$, then there is a (pwl) $\varepsilon$-isotopy $H_{t}(0 \leqq t \leqq 1)$ of $M$ onto itself such that $H_{0}=I$ (Identity), $H_{t}=I$ on $M-N(M, f(K), \varepsilon)$, and $H_{1} f_{0}=f_{1}$, and

(b) if $K$ is a 2-sphere and if $f_{0}$ and $f_{1}$ are homeomorphisms of $K$ onto disjoint tame sets in $M$ so that $d\left(f, f_{e}\right)<\delta(e=0,1)$, then there is a homeomorphism $g$ of $K \times[0,1]$ into $M$ such that $g(x, e)=$ $f_{e}(x)(x \in K, e=0,1)$ and $\rho(f(x), g(x, t))<\varepsilon(x \in K, t \in[0,1])$.

We wish to thank Dean Montgomery for pointing out certain elementary facts about equivalences of involutions.

2. Converting isotopies of $\Sigma$ and crumpled cubes into paths in $\mathscr{F}$. Here we introduce an isotopy construction to be used in $\S 6$. We omit proofs of Lemmas 2.1 and 2.2.

Lemma 2.1. Suppose $f \in \mathscr{F}(S)$ and $S$ bounds crumpled cubes $C$ and $D$ in $\Sigma$.

Then the following statements hold:

(1) If $g \in \mathscr{F}(S)$ with $g|C=f| C$, then $g=f$,

(2) If $h$ is a homeomorphism of $\Sigma$, then $h f h^{-1} \in \mathscr{F}(h(S))$, and

(3) If $g \in \mathscr{F}(S)$ and $h$ is a homeomorphism of $\Sigma$ given by $h \mid C=g f$ and $h \mid D=I$, then $g=h f h^{-1}$.

Lemma 2.2. Suppose $f \in \mathscr{F}(S)$ and $H_{t}(0 \leqq t \leqq 1)$ is an isotopy of $\Sigma$. 
Then $h_{t}(0 \leqq t \leqq 1)$ given by $h_{t}=H_{t} f H_{t}^{-1}$ is a path in $\mathscr{F}$ with $h_{t} \in \mathscr{F}\left(H_{t}(S)\right)(t \in[0,1])$.

\section{Lemma 2.3. Suppose $f \in \mathscr{F}$ and $\varepsilon>0$.}

There is a $\delta>0$ such that if $g \in N(\mathscr{F}, f, \delta)$ and $H_{t}(0 \leqq t \leqq 1)$ is a $\delta$-isotopy of $\Sigma$ with $H_{0}=I$, then $h_{t}(0 \leqq t \leqq 1)$ given by $h_{t}=H_{t} g H_{t}^{-1}$ is a path in $N(\mathscr{F}, f, \varepsilon)$.

Proof. Choose $\delta<\varepsilon / 3$ so that the image under $f$ of each $\delta$-subset of $\Sigma$ has diameter less than $\varepsilon / 3$.

Let $g$ and $H_{t}$ be given as in the hypothesis. For each $x \in \Sigma$, $t \in[0,1]$, dia $\left(x \cup H_{t}^{-1}(x)\right)<\delta$ so $g\left(x \cup H_{t}^{-1}(x)\right) \subseteq N\left(\Sigma, f\left(x \cup H_{t}^{-1}(x)\right), \delta\right) \subseteq$ $N(\Sigma, f(x), \varepsilon / 3+\delta)$ and

$$
H_{t}\left(g\left(x \cup H_{t}^{-1}(x)\right)\right) \subseteq N(\Sigma, f(x), \varepsilon / 3+2 \delta) \subseteq N(\Sigma, f(x), \varepsilon) .
$$

Thus $\rho\left(f(x), h_{t}(x)\right)<\varepsilon(x \in \Sigma, t \in[0,1])$ and $h_{t}(0 \leqq t \leqq 1) \subseteq N(\mathscr{F}, f, \varepsilon)$.

3. $\mathscr{C}$ is dense in $\mathscr{F}$. Bing and $\mathrm{Wu}[3,9,22]$ have shown that there are uncountably many inequivalent involutions in $\mathscr{F}-\mathscr{G}$. (See also [2].) In fact Bing's methods in [9, §2] can be used to show that $\mathscr{F}-\mathscr{G}$ is dense in $\mathscr{F}$. We use Bing's approximation theorem for spheres to show that $\mathscr{P}$ is also dense in $\mathscr{F}$.

TheOREM 3.1. Suppose $f \in \mathscr{F}(S)$ and $\varepsilon>0$.

There is a $\delta>0$ such that if $R$ is a tame 2-sphere in $\Sigma$ homeomorphically within $\delta$ of $S$, then there is an element $g \in \mathscr{F}(R)$ such that $d(f, g)<\varepsilon$.

Proof. Let $\varepsilon_{1}>0$ be sufficiently small so that $\varepsilon_{1}<\varepsilon$ and $d(f, f h)<\varepsilon$ for every $\varepsilon_{1}$-homeomorphism $h$ of $\Sigma$. Let $\phi$ be a homeomorphism of a polyhedral 2 -sphere $K$ onto $S$. Let $\varepsilon_{2}<\varepsilon_{1}$ correspond to $\delta$ in Theorem 1.1 for the substitution $\left(\Sigma \rightarrow M, K \rightarrow K, \phi \rightarrow f, \varepsilon_{1} \rightarrow \varepsilon\right)$. Choose a positive number $\delta<\varepsilon_{2} / 2$ so small that $\rho(x, f(x))<\varepsilon_{2} / 2(x \in N(\Sigma, S, \delta))$.

Let $R$ be a tame 2 -sphere homeomorphically within $\delta$ of $S$. There is a homeomorphism $\phi_{1}$ of $K$ onto $R$ such that $d\left(\phi, \phi_{1}\right)<\delta$. Set $\phi_{0}=f \phi_{1}$. From the conditions on $\delta$ we have $d\left(\phi_{1}, \phi_{0}\right)<\varepsilon_{2} / 2$ so $d\left(\phi, \phi_{0}\right)<\varepsilon_{2}$. From Theorem 1.1 there is an $\varepsilon_{1}$-isotopy $H_{t}(0 \leqq t \leqq 1)$ of $\Sigma$ such that $H_{0}=I$ and $H_{1} \phi_{0}=\phi_{1}$. That is, $H_{1} f \mid R=I$. Now $R$ bounds 3-cells $C$ and $D$, and $H_{1} f$ switches these 3 -cells.

Define $g$ by $H_{1} f$ on $D$ and by $f H_{1}^{-1}$ on $C$. Clearly $g \in \mathscr{F}(R)$. For $x \in D$ we have $\rho\left(f(x), H_{1} f(x)\right)<\varepsilon_{1}<\varepsilon$. For $x \in C$ we have

$$
\rho\left(f(x), f H_{1}^{-1}(x)\right)<\varepsilon
$$

by the definition of $\varepsilon_{1}$. 
From [10, Th. 1.1] we get:

Corollary 3.1. The subspace $\mathscr{G}$ is dense in $\mathscr{F}$.

4. Homeomorphic closeness of fixed point sets.

Lemma 4.1. Suppose $f \in \mathscr{F}(S)$ and $\varepsilon>0$.

There is a $\delta>0$ such that if $g \in \mathscr{F}(R)$ with $d(f, g)<\delta$ then (i) $R \cong N(\Sigma, S, \varepsilon)$, (ii) $R$ separates two points of $\Sigma-N(\Sigma, S, \varepsilon)$ if and only if $S$ separates them, and (iii) dia $(R)>\operatorname{dia}(S)-\varepsilon$.

Proof. Let $S$ separate $\Sigma$ into components $U$ and $V$. Let $Y_{1}, Z_{1}$ be nonempty, compact subsets of $U, V$ such that $\Sigma-N(\Sigma, S, \varepsilon) \cong Y_{1} \cup Z_{1}$ and for each $x \in S, N(\Sigma, x, \varepsilon / 6) \cap Y_{1} \neq \varnothing, N(\Sigma, x, \varepsilon / 6) \cap Z_{1} \neq \varnothing$. Set $\varepsilon_{1}=\inf \left\{\rho(x, f(x)) \mid x \in Y_{1} \cup Z_{1}\right\}$. Choose $\varepsilon_{2}>0$ so that

$$
\sup \left\{\rho(x, f(x)) \mid x \in N\left(\Sigma, S, \varepsilon_{2}\right)\right\}<\varepsilon_{1} / 4 .
$$

Let $Y, Z$ be compact, connected subsets of $U, V$ such that

$$
\left(\Sigma-N\left(\Sigma, S, \varepsilon_{2}\right)\right) \subseteq Y \cup Z \text {. }
$$

Set $\delta=1 / 4 \inf \{\rho(x, f(x)) \mid x \in Y \cup Z\}$.

Let $g \in \mathscr{F}(R)$ with $d(f, g)<\delta$. For each $x \in Y \cup Z, \rho(x, g(x)) \geqq$ $4 \delta-\delta$. Thus $R \subseteq \Sigma-(Y \cup Z) \subseteq N\left(\Sigma, S, \varepsilon_{2}\right) \subseteq N(\Sigma, S, \varepsilon)$. Now $R$ bounds crumpled cubes $C$ and $D$ with $Y \subseteq C$, and $g$ switches $C$ and $D$. Suppose $R$ does not separate $Y$ from $Z$. Then

$$
Y \cup Z \subseteq C, D \subseteq N\left(\Sigma, S, \varepsilon_{2}\right),
$$

and $\rho(x, g(x))<\varepsilon_{1} / 4+\delta<\varepsilon_{1} / 2(x \in D)$. Let $c \in Y_{1} \cup Z_{1}$, and let $d=$ $g(c) \in D$. Then $c=g(d)$, and we have $\rho(c, d) \geqq \varepsilon_{1}-\delta \geqq 3 / 4 \varepsilon_{1}$ because $c \in Y_{1} \cup Z_{1}$, but $\rho(d, c)<\varepsilon_{1} / 2$ because $d \in D$. From the contradiction we conclude that $R$ separates $Y$ from $Z$. Thus $R$ separates two points of $\Sigma-N(\Sigma, S, \varepsilon)$ if and only if $S$ separates them.

Let $p, q$ be points of $S$ such that $\rho(p, q)=\operatorname{dia}(S)$, and let $y_{1}, y_{2} \in Y$, $z_{1}, z_{2} \in Z$ be points such that $\rho\left(p, y_{1} \cup z_{1}\right)<\varepsilon / 6, \rho\left(q, y_{2} \cup z_{2}\right)<\varepsilon / 6$. We have $\rho\left(y_{1}, z_{1}\right)<\varepsilon / 3, \rho\left(y_{2}, z_{2}\right)<\varepsilon / 3$. Since $R$ separates $Y$ from $Z$ there are points $p^{\prime}, q^{\prime}$ of $R$ on the shorter segments of the great circles through $y_{1}, z_{1}$ and $y_{2}, z_{2}$. We have $\rho\left(p, p^{\prime}\right)<\varepsilon / 2, \rho\left(q, q^{\prime}\right)<\varepsilon / 2$ so $\operatorname{dia}(R) \geqq \rho\left(p^{\prime}, q^{\prime}\right)>\operatorname{dia}(S)-\varepsilon$.

Lemma 4.2. Suppose $f \in \mathscr{F}(S)$ and $\varepsilon>0$.

There is $a \delta>0$ such that if $g \in \mathscr{F}(R)$ with $d(f, g)<\delta$, then every simple closed curve of diameter less than $\delta$ on $R$ bounds an $\varepsilon$-disk on $R$. 
Proof. We suppose dia $(S)>4 \varepsilon$. Choose $\varepsilon_{1}>0$ so that every $5 \varepsilon_{1}$-subset of $\Sigma$ is contained in the interior of a 3-cell of diameter less than $\varepsilon / 3$. Choose $\varepsilon_{2}>0$ so that $\rho(x, f(x))<\varepsilon_{1}\left(x \in N\left(\Sigma, S, \varepsilon_{2}\right)\right)$. Choose $\varepsilon_{3}>0$ so that $\varepsilon_{3}<\varepsilon_{2} / 2$ and

$$
\varepsilon_{3}<\inf \left\{\rho(x, f(x)) \mid x \in \Sigma-N\left(\Sigma, S, \varepsilon_{2}\right)\right\} .
$$

Choose $\delta$ from Lemma 4.1 for $f$ and $\varepsilon_{3}$ sufficiently small so that every $3 \delta$-subset of $\Sigma$ is contained in the interior of a 3-cell of diameter less than $\varepsilon_{2} / 2$.

Let $g \in \mathscr{F}(R)$ with $d(f, g)<\delta$, and let $J$ be a simple closed curve on $R$ with $\operatorname{dia}(J)<\delta$. Use Theorem 3.1 and [10, Th. 1.1] to get a tame 2-sphere $R^{\prime}$ in $\Sigma$, a $\delta$-homeomorphism $\phi$ of $R$ onto $R^{\prime}$, and an element $g^{\prime} \in \mathscr{F}\left(R^{\prime}\right)$ such that $d\left(f, g^{\prime}\right)<\delta$. From Lemma 4.1, dia $\left(R^{\prime}\right)>3 \varepsilon$. Use $[4,6,19]$ to give $\Sigma$ a triangulation $T$ in which $R^{\prime}$ is a polyhedron. The set $J^{\prime}=\phi(J)$ has diameter less than $3 \delta$ and so lies in the interior of a 3-cell $C$ of diameter less than $\varepsilon_{2} / 2$. From [6] we can suppose that $\mathrm{Bd}(C)$ is a polyhedron in $T$ and is in general position with respect to $R^{\prime}$. Each component of $\mathrm{Bd}(C) \cap R^{\prime}$ is a simple closed curve which we claim bounds an $\varepsilon / 3$-disk on $R^{\prime}$.

Let $L$ be a component of $\mathrm{Bd}(C) \cap R^{\prime}$. Now $L$ bounds an $\varepsilon_{2} / 2$-disk $D$ on $\mathrm{Bd}(C)$. Let $D_{1}, \cdots, D_{m}$ denote the closures of the components of Int $(D)-R^{\prime}$. From Lemma 4.1, $R^{\prime} \subseteq N\left(\Sigma, S, \varepsilon_{3}\right)$ so $D \leqq N\left(\Sigma, S, \varepsilon_{2}\right)$, $\rho\left(x, g^{\prime}(x)\right)<\varepsilon_{1}+\delta(x \in D)$, and dia $\left(D \cup g^{\prime}(D)\right)<\varepsilon_{2}+2 \varepsilon_{1}+2 \delta<5 \varepsilon_{1}$. From the choice of $\varepsilon_{1}, D \cup g^{\prime}(D)$ is contained in an open 3-cell $U$ of diameter less than $\varepsilon / 3$. Because Int $(D)$ is in general position with respect to $R^{\prime}$, each $D_{i} \cup g^{\prime}\left(D_{i}\right)$ is a surface which bounds a 3-manifold $Q_{i}$ in $U$. Furthermore $Q_{i} \cap R^{\prime}$ is a surface $F_{i}$ whose boundary is $\operatorname{Bd}\left(D_{i}\right)$. We show that $L$ is contained in a disk in $R^{\prime} \cap U$.

Some $D_{i}$, say $D_{k}$, is a disk so $Q_{k}$ is a 3 -cell and $F_{k}$ is a disk. Either $L \leqq F_{k}$ or $L \cap F_{k}=\varnothing$. In the first case $L$ bounds a subdisk of $F_{k}$ in $R^{\prime}$ which has diameter less than $\varepsilon / 3$. In the second case locate an inner most simple closed curve $L_{j}$ of $D \cap F_{k}$ in $F_{k}$, cut out the disk $D_{k j}$ it bounds in $D$, replace that disk by the disk $F_{k j}$ which $L_{j}$ bounds in $F_{k}$, and push a neighborhood of $F_{k j}$ in the adjusted disk slightly to one side of $R^{\prime}$ to obtain a new polyhedral disk $D(1)$ with boundary $L$ such that Int $(D(1))$ is in general position with respect to $R^{\prime}$, Int $(D(1)) \cap R^{\prime}$ consists of a proper subcollection of the simple closed curves of Int $(D) \cap R^{\prime}$, and $D(1) \cup g^{\prime}(D(1)) \subseteq U$. After a sufficient number of repetitions of this process we arrive at a polyhedral disk $D(n)$ such that $D(n) \cap R^{\prime}=\mathrm{Bd}(D(n)), D(n) \cup g^{\prime}(D(n))$ bounds a 3-manifold $P$ in $U$, and $L \leqq P$. But then $F(n)=R^{\prime} \cap P$ is an $\varepsilon / 3$-disk, and $L$ bounds a subdisk of $F(n)$ in $R^{\prime}$.

Let $K_{1}, \cdots, K_{r}$ denote the $\varepsilon / 3$-disks on $R^{\prime}$ which the simple closed curves on $\mathrm{Bd}(C) \cap R^{\prime}$ bound. Since dia $\left(C \cup\left(\cup K_{i}\right)\right)<\varepsilon$ and $\operatorname{dia}\left(R^{\prime}\right)>$ 
$3 \varepsilon$, the set $R^{\prime}-\cup K_{i}$ is connected, has diameter greater than $\varepsilon$, and thus does not intersect $C$. This shows that $J^{\prime}$ is contained in one of the disks $K_{i}$ and so bounds an $\varepsilon / 3$-disk $K$ on $R^{\prime}$. Then $J$ bounds the $\varepsilon / 3+2 \delta<\varepsilon$-disk $\phi^{-1}(K)$ on $R$.

Lemma 4.3. Suppose $f \in \mathscr{F}(S), A$ is a tame arc in $\Sigma$ which pierces $S$ at a point $p$ and otherwise fails to intersect $S$, and $\varepsilon>0$.

There is an $\eta>0$ such that if $C$ is a tame 3-cell of diameter less than $\eta$ whose interior contains $p$ and which intersects $A$ in an unknotted arc $A^{\prime}$ that spans $\mathrm{Bd}(C)$, then the following statement is valid:

There is a $\delta>0$ such that if $g \in \mathscr{C}(R)$ with $d(f, g)<\delta$ and $R \cap \mathrm{Bd}(C)$ is a finite collection of simple closed curves at which $R$ crosses $\mathrm{Bd}(C)$, then $R \cap A \subseteq \operatorname{Int}\left(A^{\prime}\right)$, there is a component $U$ of $R-C$ such that each component of $R-U$ is an $\varepsilon$-disk, and exactly one component of $\mathrm{Cl}(U) \cap \mathrm{Bd}(C)$ separates the two endpoints of $\mathrm{Bd}\left(A^{\prime}\right)$ on $\operatorname{Bd}(C)$.

Proof. We suppose dia $(S) \geqq 4 \varepsilon$. Complete $A$ to a tame, unknotted simple closed curve $L$ in $\Sigma$. Let $\varepsilon_{1}<\varepsilon$ be a positive number such that $N\left(\Sigma, p, 2 \varepsilon_{1}\right) \cap L \leqq A$. Let $\eta$ correspond to $\delta$ in Lemma 4.2 for the substitution $f \rightarrow f, \varepsilon_{1} \rightarrow \varepsilon$.

Let $C$ be a tame 3-cell with the properties described in the hypothesis of the lemma. Let $\varepsilon_{2}>0$ be a number such that

$$
N\left(\Sigma, S, 5 \varepsilon_{2}\right) \cap A \subseteq \operatorname{Int}\left(A^{\prime}\right)
$$

where $A^{\prime}=C \cap A$ and $N\left(\Sigma, p, 5 \varepsilon_{2}\right) \subseteq \operatorname{Int}(C)$. Choose $\varepsilon_{3}>0$ less than $\varepsilon_{2}$ so that $\rho(x, f(x))<\varepsilon_{2}\left(x \in N\left(\Sigma, S, \varepsilon_{3}\right)\right)$. Choose $\varepsilon_{4}>0$ so that $\varepsilon_{4}<\varepsilon_{3}$ and $N\left(\Sigma, S, \varepsilon_{4}\right) \cap A$ is contained in an arc $A^{\prime \prime}$ in $N\left(\Sigma, p, \varepsilon_{3}\right)$. Choose $\delta<\varepsilon_{4}$ from Lemma 4.1 for $f, \varepsilon_{4}$.

Let $g$ be given as in the hypothesis of the lemma. It follows from the conditions on $\eta$ that each component of $R \cap \mathrm{Bd}(C)$ bounds an $\varepsilon_{1}$-disk on $R$. By throwing away disks contained in the interiors of others we find mutually exclusive $\varepsilon_{1}$-disks $F_{1}, \cdots, F_{m}$ such that each $\mathrm{Bd}\left(F_{i}\right) \subseteq \mathrm{Bd}(C)$ and $R \cap \mathrm{Bd}(C) \subseteq \bigcup F_{i}$. From the conditions on $\varepsilon_{1}$ no $F_{i}$ intersects $L-A$, and from the conditions on $\delta, R \cap A \subseteq \operatorname{Int}\left(A^{\prime}\right)$. Set $U=R-\bigcup F_{i}$. Now $U$ is connected so either $U \subseteq \operatorname{Int}(C)$ or $U \cap C=\varnothing$. In the first case $\operatorname{dia}(R)<2 \varepsilon_{1}+\eta<3 \varepsilon_{1}<\operatorname{dia}(S)-\varepsilon$. But from the choice of $\delta$, dia $(R)>\operatorname{dia}(S)-\varepsilon$ so $U \cap C=\varnothing$. Because $A$ pierces $S$ the endpoints of $A^{\prime}$ lie in different components of $\Sigma-S$. Lemma 4.1 shows that the endpoints of $A^{\prime}$ also lie in different components of $\Sigma-R$.

Suppose no $\mathrm{Bd}\left(F_{i}\right)$ separates the endpoints of $A^{\prime}$ on $\mathrm{Bd}(C)$. From 
[7, Th. 7.3] there is a homeomorphism of $\Sigma$ onto itself which is the identity on $L-A^{\prime}$ and pulls $R$ entirely off $C$. But this contradicts the fact that $R$ separates the endpoints of $A^{\prime}$. Suppose $\mathrm{Bd}\left(F_{i}\right)$ and $\mathrm{Bd}\left(F_{j}\right)(i \neq j)$ both separate the endpoints of $A^{\prime}$ on $\mathrm{Bd}(C)$. Both $\mathrm{Bd}\left(F_{i}\right)$ and $\mathrm{Bd}\left(F_{j}\right)$ link $L$, and both $F_{i}$ and $F_{j}$ fail to intersect $L-\operatorname{Int}\left(A^{\prime}\right)$ so they both intersect Int $\left(A^{\prime}\right)$. Thus there are distinct components $H$ and $K$ of $R \cap C$ and there is a subarc $B$ of $A^{\prime}$ with endpoints $r \in H$ and $s \in K$ so that Int $(B)$ fails to intersect $R$. The conditions on $\delta$ show that $B \subseteq A^{\prime \prime} \subseteq N\left(\Sigma, p, \varepsilon_{3}\right)$. Furthermore

$$
\rho(x, g(x)) \leqq \delta+\varepsilon_{2} \leqq 2 \varepsilon_{2}\left(x \in N\left(\Sigma, p, \varepsilon_{3}\right)\right)
$$

so $g\left(A^{\prime \prime}\right) \subseteq N\left(\Sigma, p, 5 \varepsilon_{2}\right) \subseteq \operatorname{Int}(C)$. Now $H$ separates $C$ into components $U$ and $V$. Suppose $K \subseteq U$. Because $g$ switches the 3-cells bounded by $R, g(B-R) \subseteq V$. But this is nonsense for $g(s)=s$. From the contradiction we conclude that exactly one $\mathrm{Bd}\left(F_{i}\right)$ separates the endpoints of $A^{\prime}$ on $\mathrm{Bd}(C)$.

The following lemma is essentially Theorem 6.1 of [11]. It is obtained by changing $E^{3}$ to $\Sigma$, the disk $D$ to a 2 -sphere $S$, introducing a triangulation of $\Sigma$, and making a few small adjustments in the proof of the theorem-one of them is pushing the triangulation of $\Sigma$ keeping $S$ fixed rather than the other way around.

Lemma 4.4. Suppose $S$ is a 2-sphere in $\Sigma$ and $\varepsilon>0$.

There is a triangulation $T$ of $\Sigma$ with $i$-skeleton $T_{i}$ and mesh less than $\varepsilon$, there is a tame Sierpinski curve $X$ on $S$, and there is an $\varepsilon$-homeomorphism $g$ of $S$ onto a tame sphere $S^{\prime}$ so that

(1) each component of $S-X$ has diameter less than $\varepsilon$,

(2) $g$ is the identity on $X$,

(3) $S$ misses $T_{0}$ and $S \cap T_{1}$ is a finite collection of points in $I(X, S)$ (the inaccessible points of $X$ in $S$ ) where 1-simplexes of $T$ pierce $S$,

(4) $g(S)$ is a polyhedron in $T$ which is in general position with respect to $T_{2}$, and

( 5 ) $g(S) \cap T_{2}=X \cap T_{2}=I(X, S) \cap T_{2}$.

Following [11] we say, for a 2-sphere $S$ in $\Sigma$, a tame Sierpinski curve $X$ in $S$, and a triangulation $T$ of $\Sigma$ with $i$-skeleton $T_{i}$ and mesh less than $\varepsilon,\left(S, X, T_{2}, \varepsilon\right)$ has Property $Q$ provided there is an $\varepsilon$-homeomorphism $g$ of $S$ onto a tame sphere $S^{\prime}$ so that the five conditions are satisfied in the conclusion of Lemma 4.4.

Bing [7] defines a stable graph as a finite, planar graph such that each homeomorphism between two embeddings of it into 2-spheres can 
be extended to a homeomorphism between the 2-spheres. The following lemma about stable graphs is similar to Theorem 3.2 of [7].

Lemma 4.5. Suppose $f \in \mathscr{F}(S)$ and $\varepsilon>0$.

There is a stable graph $G=\mathrm{U} \mathrm{Bd}\left(D_{i}\right)$ where $D_{1}, \cdots, D_{m}$ are $\varepsilon$ disks filling up $S$ and having mutually exclusive interiors, and there is a $\delta>0$ such that if $g \in \mathscr{F}(R)$ with $d(f, g)<\delta$, then there is an $\varepsilon$-homeomorphism $\pi$ of $G$ into $R$.

Proof. From Lemma 4.4 and $[7, \S 9]$ it follows as in [12, Lemmas 6.2 and 6.3] that there is a triangulation $T$ of $\Sigma$ with $i$-skeleton $T_{i}$, a tame Sierpinski curve $X$ on $S$, and an $\varepsilon_{1}>0$ so that $\left(S, X, T_{2}, \varepsilon_{1}\right)$ has Property $Q$, and if $G^{\prime}$ denotes the graph which consists of the sum of the components of $X \cap T_{2}$ containing points of $T_{1}$, then $G^{\prime}$ contains a stable subgraph $G=\mathrm{U} B d\left(D_{i}\right)$ where $D_{1}, \cdots, D_{m}$ are $\varepsilon / 3$-disks filling up $S$ and having mutually exclusive interiors. Let $t_{1}, \cdots, t_{j}, \cdots$ denote the arcs which are the closures of the components of $G-T_{1}$, and let $p_{1}, \cdots, p_{k}, \cdots$ denote the points of $G \cap T_{1}$.

Let $\varepsilon_{2}>0$ be so small that each dia $\left(N\left(\Sigma, D_{i}, \varepsilon_{2}\right)\right)<\varepsilon / 3$. Because the accessible points of $X$ fail to intersect $T_{2}$ there is a homeomorphism $\lambda$ of $G \times[-1,1]$ into $T_{2}$ so that (1) for each simplex $s$ of $T$,

$$
\lambda((\operatorname{Int}(s) \cap G) \times[-1,1]) \leqq \operatorname{Int}(s),
$$

(2) $\lambda\left(\left(G \cap T_{1}\right) \times[-1,1]\right) \cap G=G \cap T_{1}$, (3) for each $D_{i}$,

$$
\lambda\left(\operatorname{Bd}\left(D_{i}\right) \times[-1,1]\right) \subseteq N\left(\Sigma, D_{i}, \varepsilon_{2}\right),
$$

and (4) $G_{-1}=\lambda(G \times-1)$ and $G_{1}=\lambda(G \times 1)$ lie in different components of $\Sigma-S$. One obtains $G_{-1}, G_{1}$ satisfying (4) in much the same way that one finds the piercing arcs in $[8, \S 4]$. For each $p_{k}$ set $A_{k}=$ $\lambda\left(p_{k} \times[-1,1]\right)$.

Choose $\varepsilon_{3}>0$ so that $\varepsilon_{3}<\varepsilon_{2}, N\left(\Sigma, S, \varepsilon_{3}\right) \cap\left(G_{-1} \cup G_{1}\right)=\varnothing$, the sets $N\left(\Sigma, p_{k}, \varepsilon_{3}\right)$ are mutually exclusive, and each $N\left(\Sigma, p_{k}, \varepsilon_{3}\right) \cap T_{1} \subseteq \operatorname{Int}\left(A_{k}\right)$. Choose $\varepsilon_{4}<\varepsilon_{3} / 2$ so that it corresponds to $\eta$ in Lemma 4.3 for $f$ and $\varepsilon_{3} / 2$. For each $p_{k}$ let $C_{k}$ be a 3-cell of diameter less than $\varepsilon_{4}$ whose interior contains $p_{k}$, which is polyhedral in $T$ and in general position with respect to $T_{2}$, and whose intersection with each simplex $s$ of $T$ is either empty or a cell of the dimension of $s$. For each $C_{k}$ let $A_{k}^{\prime}$ denote the subarc $C_{k} \cap T_{1}$ of $A_{k}$. Finally choose $\delta$ so that it is subject to the conditions on $\delta$ in Lemma 4.1 for $f$ and $\varepsilon_{3}$ and subject to the conditions on $\delta$ in Lemma 4.3 for each substitution $\left(f \rightarrow f, A_{k} \rightarrow A\right.$, $\left.C_{k} \rightarrow C, \varepsilon_{3} / 2 \rightarrow \varepsilon\right)$.

Let $g \in \mathscr{F}(R)$ with $d(f, g)<\delta$. Use [5, Th. 7] and Theorem 3.1 to find a polyhedral 2-sphere $R^{\prime}$ in $T$ which is in general position with 
respect to $T_{2}$ and each $C_{k}$ so that there is a $\delta$-homeomorphism $\phi$ of $R$ onto $R^{\prime}$ and an involution $g^{\prime} \in \mathscr{F}\left(R^{\prime}\right)$ with $d\left(f, g^{\prime}\right)<\delta$.

From the conditions on $\delta, \varepsilon_{2}$, and $\varepsilon_{3}$ we find that (1) $G_{-1}$ and $G_{1}$ lie in different components of $\Sigma-R^{\prime}$, (2) each $A_{k} \cap R^{\prime} \subseteq \operatorname{Int}\left(A_{k}^{\prime}\right)$, (3) there is a component $U$ of $R^{\prime}-\bigcup C_{k}$ such that each component of $R^{\prime}-U$ is an $\varepsilon_{3} / 2$-disk - denote these disks by $F_{k j}$ so that $\mathrm{Bd}\left(F_{k j}\right) \subseteq$ $\mathrm{Bd}\left(C_{k}\right)$-and (4) for each $k$ exactly one $\mathrm{Bd}\left(F_{k j}\right)$, say $\mathrm{Bd}\left(F_{k 1}\right)$, separates the endpoints of $A_{k}^{\prime}$ on $\mathrm{Bd}\left(C_{k}\right)$.

Following Step 2 in $\S 4$ of [7] we define a homeomorphism $h$ of $\Sigma$ which is the identity on $\mathrm{Cl}(U) \cup\left(T_{1}-\bigcup C_{k}\right) \cup\left(\Sigma-\bigcup N\left(\Sigma, p_{k}, \varepsilon_{3}\right)\right)$ so that each $h\left(F_{k j}\right)(j>1)$ fails to intersect $T_{1}$ and each $h\left(\operatorname{Int}\left(F_{k 1}\right)\right) \subseteq$ Int $\left(C_{k}\right)$. We can suppose that $h\left(R^{\prime}\right)$ is in general position with respect to $T_{2}$ and that each $h\left(F_{k 1}\right) \cap T_{1}$ is the single point $p_{k}$. Because $h\left(R^{\prime}\right)$ separates $G_{-1}$ from $G_{1}$ there must be an arc $t_{j}^{\prime}$ in each disk $\lambda\left(t_{j} \times[-1,1]\right)$ which spans $\mathrm{Bd}\left(\lambda\left(t_{j} \times[-1,1]\right)\right)$ and has

$$
\mathrm{Bd}\left(t_{j}\right)=h\left(R^{\prime}\right) \cap \mathrm{Bd}\left(\lambda\left(t_{j} \times[-1,1]\right)\right)
$$

for its endpoints. Define a homeomorphism $\pi^{\prime}$ of $G$ into $h\left(R^{\prime}\right)$ so that each $\pi^{\prime}\left(p_{k}\right)=p_{k}$ and each $\pi^{\prime}\left(t_{j}\right)=t_{j}^{\prime}$.

Define the homeomorphism $\pi$ by $\pi=\phi^{-1} h^{-1} \pi^{\prime}$. Each of $\pi^{\prime}, h^{-1}$, and $\phi^{-1}$ is an $\varepsilon / 3$-homeomorphism so $\pi$ is an $\varepsilon$-homeomorphism of $G$ into $R$.

THEOREM 4.6. Suppose $f \in \mathscr{F}(S)$ and $\varepsilon>0$.

There is a $\delta>0$ such that if $g \in \mathscr{F}(R)$ with $d(f, g)<\delta$ then there is an $\varepsilon$-homeomorphism of $S$ onto $R$.

Proof. We suppose dia $(S)>5 \varepsilon$. Let $3 \varepsilon_{1}<\varepsilon / 2$ correspond to $\delta$ in Lemma 4.2 for $f$ and $\varepsilon / 2$. Choose $\delta$ from Lemma 4.5 for $f$ and $\varepsilon_{1}$.

Let $g \in \mathscr{F}(R)$ with $d(f, g)<\delta$. From Lemma 4.5 there are $\varepsilon_{1}$-disks $D_{1}, \cdots, D_{m}$ which fill up $S$ and have mutually exclusive interiors so that $G=\mathrm{UBd}\left(D_{i}\right)$ is a stable graph, and there is an $\varepsilon_{1}$-homeomorphism $\pi$ of $G$ into $R$. Since $G$ is stable we can extend $\pi$ to a homeomorphism of $S$ onto $R$ which we also call $\pi$. Each $\pi\left(\operatorname{Bd}\left(D_{i}\right)\right)$ has diameter less than $3 \varepsilon_{1}$ so by the choice of $\varepsilon_{1}$ it bounds an $\varepsilon / 2$-disk $F_{i}$ in $R$. Suppose for some $D_{i}, \pi\left(D_{i}\right) \neq F_{i}$. Then $\pi(G) \subseteq F_{i}$. But dia $(G)>3 \varepsilon$ and $\pi \mid G$ is an $\varepsilon_{1}$-homeomorphism so dia $(\pi(G))>\varepsilon>\operatorname{dia}\left(F_{i}\right)$. Thus each $\pi\left(D_{i}\right)=$ $F_{i}$ and $\pi$ is a $3 \varepsilon_{1}+\varepsilon / 2<\varepsilon$-homeomorphism of $S$ onto $R$.

5. Small deformations of cells whose boundaries approximate a given sphere. We omit a proof of Lemma 5.1. The proof is straight forward but involves a tedious pasting together of small isotopies.

Lemma 5.1. Suppose $M$ is a 3-manifold, $F$ is a compact surface, $f$ is a homeomorphism of $F$ into $M$, and $\varepsilon>0$. 
There is a $\delta>0$ such that if $g$ is a homeomorphism of $F \times[0,1]$ onto a solid $P$ in $M$ where $\rho(f(x), g(x, t))<\delta(x \in F, t \in[0,1])$, and if $h$ is a $\delta$-homeomorphism of $P$ onto itself which is the identity on $\mathrm{Bd}(P)$, then there is an $\varepsilon$-isotopy $H_{t}(0 \leqq t \leqq 1)$ of $P$ onto itself such that $H_{0}=I, H_{t} \mid \mathrm{Bd}(P)=I$, and $H_{1} h=I$.

The following theorem is the key to establishing the connectivity properties of $\mathscr{F}$.

THEOREM 5.2. Suppose $S$ is a 2-sphere in $\Sigma$ and $\varepsilon>0$.

There is a $\delta>0$ such that if $C$ is a 3-cell in $\Sigma$ whose boundary $R$ is homeomorphically within $\delta$ of $S$, and if $h$ is a $\delta$-homeomorphism of $C$ onto itself which is the identity on $R$, then there is an $\varepsilon$-isotopy $H_{t}(0 \leqq t \leqq 1)$ of $C$ onto itself such that $H_{0}=I, H_{t} \mid R=I$, and $H_{1} h=I$.

Proof. If $S$ were tame the problem would be relatively easy. With the help of Theorem 1.1 and Lemma 0 of [15] we could construct the isotopy essentially as the Alexander isotopy is constructed in [1]. However, in order to deal with 2 -spheres which are possibly wild we have to reach our goal by a devious route.

It is easily seen that an equivalent theorem is obtained if in the hypothesis $\Sigma$ is replaced by $E^{3}$. It is this equivalent version which we prove. Except for item (5) we suggest that on first reading one skip the epsilonics which follow in the next paragraph and concentrate on the geometry in the proof.

Consider then a 2-sphere $S$ in $E^{3}$ and a number $\varepsilon>0$. We suppose for convenience that dia $(S)>10 \varepsilon$. Let $f$ be a homeomorphism of a polyhedral 2-sphere $K$ onto $S$. We obtain in succession seven positive numbers $-\varepsilon_{1}, \cdots, \varepsilon_{6}$, and $\delta$.

(1) Conditions on $\varepsilon_{1}$ : Substitute $\left(E^{3} \rightarrow M, K \rightarrow F, f \rightarrow f, \varepsilon / 4 \rightarrow \varepsilon\right)$ in Lemma 5.1 to get $\varepsilon_{1}>0$ corresponding to $\delta$ there.

(2) Conditions on $\varepsilon_{2}$ : Substitute $\left(E^{3} \rightarrow M, K \rightarrow K, f \rightarrow f, \varepsilon_{1} \rightarrow \varepsilon\right)$ in Theorem 1.1 to get $\varepsilon_{2}>0$ corresponding to $\delta$ there.

(3) Conditions on $\varepsilon_{3}$ : Choose $\varepsilon_{3}>0$ so that $\varepsilon_{3}<\varepsilon_{2} / 8$ and $\varepsilon_{1} / 400$.

(4) Conditions on $\varepsilon_{4}$ : Choose $\varepsilon_{4}>0$ so that every $3 \varepsilon_{4}$-subset of $S$ is contained in a disk on $S$ of diameter less than $\varepsilon_{3} / 3$.

(5) A special polyhedral neighborhood $M$ of $S$ : Use [16] to find a pwl homeomorphism $g$ of $K \times[0,1]$ onto a polyhedron $P$ in $E^{3}$ with boundary components $S_{0}=g(K \times 0)$ and $S_{1}=g(K \times 1)$ so that $\rho(g(y, e), f(y))<\varepsilon_{4}(y \in K, e=0,1)$ and to find mutually exclusive, polyhedral cubes-with-handles $G_{1}, \cdots, G_{m}, K_{1}, \cdots, K_{n}$ so that each dia $\left(G_{i}\right)<$ $\varepsilon_{4}$, each dia $\left(K_{i}\right)<\varepsilon_{4}$, each $G_{i} \cap P$ is a polyhedral disk on $S_{1}$, and each $K_{i} \cap P$ is a polyhedral disk on $S_{0}$ and so that $M=P \cup\left(\bigcup G_{i}\right) \cup\left(\bigcup K_{i}\right)$ contains a neighborhood of $S$ in $E^{3}$. We suppose that $S_{0} \subseteq \operatorname{Int}\left(S_{1}\right)$. 
Let $F$ denote the component of $\mathrm{Bd}(M)$ which intersects $S_{0}$, and let $Q$ denote the polyhedral 3-manifold which $F$ bounds in Int $(S)$.

(6) Conditions on $\varepsilon_{5}$ : Choose $\varepsilon_{5}$ less than one fourth the distance from $Q$ to $S$ and so small that any 2-sphere in $E^{3}$ which is homeomorphically within $\varepsilon_{5}$ of $S$ contains $N\left(E^{3}, Q, 4 \varepsilon_{5}\right)$ in its interior. Theorem VI 10 of [14] guarantees that the second condition can be met.

(7) Conditions on $\varepsilon_{6}$ : Substitute $\left(E^{3} \rightarrow M, Q \rightarrow K, I\right.$ (Identity) $\rightarrow$ $f, \varepsilon_{5} \rightarrow \varepsilon$ ) in Theorem 1.1 to get $\varepsilon_{6}$ corresponding to $\delta$ there.

(8) Conditions on $\delta$ : Choose $\delta<\varepsilon_{6} / 2$.

Now let $C, R$, and $h$ be given as in the hypothesis of the theorem. We construct in succession isotopies $H_{t}^{i}(0 \leqq t \leqq 1)(i=1, \cdots, 4)$ of $C$ onto itself such that each $H_{0}^{i}=I$.

Use $[6,18]$ together with $[15$, Lemma 0$]$ to obtain a $\delta$-isotopy $H_{t}^{1}$ which is the identity on $\mathrm{Bd}(C)$ so that $H_{1}^{1} h$ is locally pwl on $\operatorname{Int}(C)$. Now $H_{1}^{1} h \mid Q$ is an $\varepsilon_{6}$-homeomorphism so from items (6) and (7) there is a pw1 $\varepsilon_{5}$-isotopy $H_{t}^{2}$ of $C$ which is the identity on $\mathrm{Bd}(C)$ such that $H_{1}^{2} H_{1}^{1} h \mid Q=I$.

In each $K_{i}$ there are mutually exclusive, polyhedral $\varepsilon_{4}$-disks $D_{i j}$ spanning $\mathrm{Bd}\left(K_{i}\right)$ such that the closure of $K_{i}$ minus thin, disjoint regular neighborhoods of the $D_{i j}$ 's is a 3-cell. We can suppose that the $D_{i j}$ 's fail to intersect $S_{0}$. Use [5, Th. 7] and the fact that $R$ is collared in $C$ to find a $\delta$-homeomorphism $\theta$ of $S$ onto a polyhedral 2-sphere $R^{\prime}$ in $\operatorname{Int}(C) \cap \operatorname{Int}(M)$ which is in general position with respect to $\bigcup D_{i j}$. Each component of $\theta^{-1}\left(\left(\bigcup D_{i j}\right) \cap R^{\prime}\right)$ has diameter less than $\varepsilon_{4}+2 \delta<3 \varepsilon_{4}$ and so by (4) bounds an $\varepsilon_{3} / 3$-disk on $S$; thus each component of $\left(\bigcup D_{i j}\right) \cap R^{\prime}$ bounds a disk on $R^{\prime}$ of diameter less than $\varepsilon_{3} / 3+2 \delta<\varepsilon_{3}$. By cutting away some closures of components of $\left(D_{i j}-R^{\prime}\right)$ 's, replacing them by closures of components of $\left(R^{\prime}-\cup D_{i j}\right)$, and then pushing these modified disks slightly into Int $\left(R^{\prime}\right)$ we obtain a new collection of mutually exclusive, polyhedral $3 \varepsilon_{3}$-disks $\left\{E_{i j}\right\}$ in $M \cap \operatorname{Int}(C)$ which span $F$ and have the same boundaries as the $D_{i j}$ 's. Choose mutually exclusive regular neighborhoods $N_{i j}$ of the $E_{i j}$ 's in $M \cap \operatorname{Int}(C)$ so that each $N_{i j}$ has diameter less than $3 \varepsilon_{3}$ and intersects $\mathrm{Bd}(M)$ in a regular neighborhood of $\mathrm{Bd}\left(E_{i j}\right)$ missing $S_{0}$. Then in each $N_{i j}$ choose a smallar regular neighborhood $N_{i j}^{\prime}$ of $E_{i j}$ so that $N_{i j}^{\prime} \cap \mathrm{Bd}\left(N_{i j}\right)$ is a regular neighborhood of $\mathrm{Bd}\left(E_{i j}\right)$ in Int $\left(N_{i j} \cap \mathrm{Bd}(M)\right)$.

Now $H_{1}^{2} H_{1}^{1} h$ is a $\delta+\delta+\varepsilon_{5}<\varepsilon_{3}$-homeomorphism so each $H_{1}^{2} H_{1}^{1} h\left(N_{i j}^{\prime}\right)$ has diameter less than $5 \varepsilon_{3}$. Pushing each $H_{1}^{2} H_{1}^{1} h\left(N_{i j}^{\prime}\right) \cap \operatorname{Int}(M)$ slightly so it is in general position with respect to $U N_{i j}$ and then using [7, §7] (see also [12, Lemma 2.9]) we define a $65 \varepsilon_{3}$-isotopy $H_{t}^{3}(0 \leqq t \leqq 1)$ of $C$ so that $H_{t}^{3} \mid Q \cup \mathrm{Bd}(C)=I$ and $H_{1}^{3} H_{1}^{2} H_{1}^{1} h \mid Q \cup\left(\cup N_{i j}^{\prime}\right)=I$.

Consider the 3-manifold $T=\mathrm{Cl}\left(C-\left(Q \cup\left(\bigcup N_{i j}^{\prime}\right)\right)\right)$. Its boundary components are the 2-spheres $R=\mathrm{Bd}(C)$ and $R^{\prime \prime}$ which is obtained 
from $F$ by cutting out annuli and replacing them by pairs of disks. More specifically, for each $K_{i}, \bigcup_{j}\left(N_{\imath \jmath}^{\prime} \cap F\right)$ is replaced by

$$
\mathrm{Cl}\left(\left(\bigcup_{j} \mathrm{Bd}\left(N_{i j}^{\prime}\right)\right)-F\right) \text {. }
$$

Each dia $\left(K_{i} \cup\left(\bigcup_{j} N_{i j}^{\prime}\right)\right)<\varepsilon_{4}+2\left(3 \varepsilon_{3}\right)<7 \varepsilon_{3}$. Define a homeomorphism $f_{0}$ of $K$ onto $R^{\prime \prime}$ such that $f_{0}(y)=g(y, 0)\left(y \notin g^{-1}\left(\bigcup K_{i}\right)\right)$ and for each $K_{i}, f_{0}\left(g^{-1}\left(K_{i} \cap S_{0}\right)\right) \cong K_{i} \cup\left(\bigcup_{j} N_{i j}^{\prime}\right)$. We have $d\left(f, f_{0}\right)<\varepsilon_{4}+7 \varepsilon_{3}<8 \varepsilon_{3}<\varepsilon_{2}$. Let $f_{1}$ be a homeomorphism of $K$ onto $R$ such that $d\left(f, f_{1}\right)<\delta$.

From (2) and the fact that $R$ is collared in $C$ there is a homeomorphism $\phi$ of $K \times[0,1]$ onto $T$ such that $\phi(y, e)=f_{e}(y)(y \in K, e=0,1)$ and $\rho(\dot{\phi}(y, t), f(y))<\varepsilon_{1}(y \in K, t \in[0,1])$. Furthermore by (3) $H_{1}^{3} H_{1}^{2} H_{1}^{1} h$ is an $\varepsilon_{1}$-homeomorphism. Thus by (1) there is an $\varepsilon / 4$-isotopy $H_{t}^{4}(0 \leqq$ $t \leqq 1)$ of $C$ such that $H_{t}^{4} \mid C-\operatorname{Int}(T)=I$ and $H_{1}^{4} H_{1}^{3} H_{1}^{2} H_{1}^{1} h=I$.

The promised isotopy $H_{t}$ is given by $H_{0}=I$ and

$$
H_{t}=H_{4(t-(i-1) / 4}^{i} H_{(i-1) / 4}((i-1) / 4 \leqq t \leqq i / 4, i=1, \cdots, 4) \text {. }
$$

Each $H_{t}^{2}$ is an $\varepsilon / 4$-isotopy so $H_{t}$ is an $\varepsilon$-isotopy.

6. Pathwise and local pathwise connectivity of $\mathscr{F}$.

THeOREm 6.1. The space $\mathscr{F}^{-}$is pathwise and locally pathwise connected.

Proof. The proof is divided into four parts.

(1) The subspace $\mathscr{C}$ is locally pathwise connected at each point of $\mathscr{F}$. That is, if $f \in \mathscr{F}$ and $\varepsilon>0$, there is a $\delta>0$ such that if $g_{0}, g_{1} \in N(\mathscr{F}, f, \delta) \cap \mathscr{Y}$, then there is a path $h_{t}(0 \leqq t \leqq 1)$ in $N(\mathscr{F}, f, \varepsilon) \cap$ $\mathscr{G}$ with endpoints $h_{0}=g_{0}$ and $h_{1}=g_{1}$.

Proof of (1). Let $\varepsilon_{1}$ correspond to $\delta$ in Lemma 2.3 for $f$ and $\varepsilon$. Let $\varepsilon_{2}$ correspond to $\delta$ in Theorem 5.2 for $S$ and $\varepsilon_{1}$. Let $\varepsilon_{3}<\varepsilon_{2}$ be a positive number so small that $d\left(f_{1} f_{2}, I\right)<\varepsilon_{2}$ for each pair of elements $f_{1}, f_{2} \in N\left(\mathscr{F}, f, \varepsilon_{3}\right)$. Let $\varepsilon_{4}$ correspond to $\delta$ in Lemma 2.3 for $f, \varepsilon_{3}$. Let $\phi$ be a homeomorphism of a polyhedral 2-sphere $K$ onto $S$. Let $\varepsilon_{5}$ correspond to $\delta$ in Theorem 1.1 for the substitution $(\Sigma \rightarrow M, K \rightarrow K$, $\left.\phi \rightarrow f, \varepsilon_{4} \rightarrow \varepsilon\right)$. Finally choose $\delta<\varepsilon_{5}$ from Theorem 4.6 for $f$ and $\varepsilon_{5}$.

Let $g_{0}, g_{1} \in N(\mathscr{F}, f, \delta) \cap$ with $g_{0} \in \mathscr{C}\left(S_{0}\right)$ and $g_{1} \in \mathscr{C}\left(S_{1}\right)$. From the conditions on $\delta$ and $\varepsilon_{5}$ there are homeomorphisms $\phi_{0}, \phi_{1}$ of $K$ onto $S_{0}, S_{1}$ so that $d\left(\dot{\phi}, \phi_{e}\right)<\varepsilon_{5}(e=0,1)$ and there is an $\varepsilon_{4}$-isotopy $H_{t}^{1}(0 \leqq$ $t \leqq 1)$ of $\Sigma$ such that $H_{0}^{1}=I$ and $H_{1}^{1} \dot{\phi}_{0}=\phi_{1}$. Define $h_{t}(0 \leqq t \leqq 1 / 2)$ by $h_{t}=H_{2 t}^{1} g_{0}\left(H_{2 t}^{1}\right)^{-1}$. From Lemmas 2.2 and $2.3, h_{t}(0 \leqq t \leqq 1 / 2) \leqq$ $N\left(\mathscr{F}, f, \varepsilon_{3}\right) \cap \mathscr{G}$ and $h_{1 / 2} \in \mathscr{G}\left(S_{1}\right)$. The conditions on $\varepsilon_{3}$ show that $d\left(h_{1 / 2} g_{1}^{-1}, I\right)<\varepsilon_{2}$.

Let $S_{1}$ bound 3-cells $C$ and $D$ in $\Sigma$. From Theorem 5.2 there is 
an $\varepsilon_{1}$-isotopy $H_{t}^{2}(0 \leqq t \leqq 1)$ of $\Sigma$ onto itself such that $H_{0}^{2}=I, H_{t}^{2} \mid D=I$, and $H_{1}^{2} \mid C=g_{1} h_{1 / 2}$. Define $h_{t}(1 / 2 \leqq t \leqq 1)$ by $H_{2 t-1}^{2} h_{1 / 2}\left(H_{2 t-1}^{2}\right)^{-1}$. Lemmas 2.1 and 2.3 show that $h_{t}(1 / 2 \leqq t \leqq 1) \leqq N(\mathscr{F}, f, \varepsilon) \cap \mathscr{G}\left(S_{1}\right)$ and $h_{1}=$ $g_{1}$. Thus $h_{t}(0 \leqq t \leqq 1) \leqq N(\mathscr{F}, f, \varepsilon) \cap \mathscr{G}$ with $h_{0}=g_{0}$ and $h_{1}=g_{1}$.

(2) If $f \in \mathscr{F}$ and $\varepsilon>0$ there is a path $h_{t}(0 \leqq t \leqq 1)$ in $N(\mathscr{F}, f, \varepsilon)$ such that $h_{1}=f$ and $h_{t} \in \mathscr{G}(t<1)$.

Proof of (2). From (1) there is for each $j \geqq 1$ a $\delta_{j}$ such that any two points in $N\left(\mathscr{F}, f, \delta_{j}\right) \cap \mathscr{G}$ can be joined by a path in $N(\mathscr{F}, f, \varepsilon / j) \cap$ $\mathscr{G}$. We suppose that $\delta_{1}>\delta_{2}>\cdots>\delta_{n}>\cdots$. Use Corollary 3.1 to find for each $j$ an element $f_{j} \in N\left(\mathscr{F}, f, \delta_{j}\right) \cap \mathscr{G}$. For each $j$ there is a path $h_{t}(1-1 / j \leqq t \leqq 1-1 /(j+1))$ in $N(\mathscr{F}, f, \varepsilon / j) \cap \mathscr{G}$ such that $h_{1-1 / j}=f_{j}$ and $h_{1-1 /(j+1)}=f_{j+1}$. Because $\lim f_{j}=f$ we can set $h_{1}=f$ to get the promised path.

(3) The space $\mathscr{F}$ is locally pathwise connected.

Proof of (3). Let $f \in \mathscr{F}$ and $\varepsilon>0$. Choose $\delta$ from (1) for $f$ and $\varepsilon$.

Let $f_{0}, f_{1} \in N(\mathscr{F}, f, \delta)$. From (2) there are paths $h_{t}(0 \leqq t \leqq 1 / 4)$ and $h_{t}(3 / 4 \leqq t \leqq 1)$ in $N(\mathscr{F}, f, \delta)$ such that $h_{0}=f_{0}, h_{1}=f_{1}$, and $h_{1 / 4}$, $h_{3 / 4} \in \mathscr{G}$. Then (1) enables us to define $h_{t}(1 / 4 \leqq t \leqq 3 / 4)$ in $N(\mathscr{F}, f, \varepsilon)$ so it connects up $h_{1 / 4}$ and $h_{3 / 4}$.

(4) The space $\mathscr{F}$ is pathwise connected.

Proof of (4). In view of (2) it is sufficient to show that $\mathscr{C}$ is pathwise connected.

Let $f, g \in \mathscr{G}$ with $f \in \mathscr{G}(S)$ and $g \in \mathscr{C}(R)$. Let $T$ be a tame 2sphere in $\Sigma$ disjoint from both $S$ and $R$ so that $T$ bounds a 3-cell $B$ containing both $S$ and $R$. The pairs $(T, S)$ and $(T, R)$ both bound annuli and $S, R$ bound 3-cells $C, D$ in $\operatorname{Int}(B)$. Thus there is a homeomorphism $h$ of $B$ onto itself which is the identity on $\mathrm{Bd}(B)$ and takes $C$ onto $D$. From [1] there is an isotopy $H_{t}^{1}(0 \leqq t \leqq 1)$ of $\Sigma$ which is the identity on $\Sigma-\operatorname{Int}(B)$ so that $H_{0}^{1}=I$ and $H_{1}^{1} \mid B=h$. From Lemma $2.2, h_{t}(0 \leqq t \leqq 1 / 2)$ given by $h_{t}=H_{2 t}^{1} f\left(H_{2 t}^{1}\right)^{-1}$ is a path in $\mathscr{G}$ with $h_{0}=f$ and $h_{1 / 2} \in \mathscr{G}(R)$.

Use [1] to define an isotopy $H_{t}^{2}(0 \leqq t \leqq 1)$ of $\Sigma$ onto itself such that $H_{0}^{2}=I, H_{t}^{2} \mid \Sigma-D=I$, and $H_{1}^{2} \mid D=g h_{1 / 2}$. Define $h_{t}(1 / 2 \leqq t \leqq 1)$ by $h_{t}=H_{2 t-1}^{2} h_{1 / 2}\left(H_{2 t-1}^{2}\right)^{-1}$. As in the proof of (1) $h_{t}$ is a path in $\mathscr{G}$ with $h_{1}=g$.

The following corollary shows that pseudo isotopies like the one Bing uses in [3] can be used to obtain all elements of $\mathscr{F}-\mathscr{G}$ from $\mathscr{G}$.

CoRollary 6.1. For each pair of involutions $f \in \mathscr{F}, g \in \mathscr{G}$ there is a pseudo isotopy $H_{t}(0 \leqq t \leqq 1)$ of $\Sigma$ such that $H_{0}=I$ and $f=H_{1} g\left(H_{1}\right)^{-1}$.

Proof. Let $f \in \mathscr{F}$ and $g \in \mathscr{G}$ be given. From (2) there is an 
element $g_{2} \in \mathscr{G}$ and there is a path $h_{t}(1 / 2 \leqq t \leqq 1)$ in $\mathscr{F}$ such that $h_{1 / 2}=g_{2}$ and $h_{1}=f$. From the proof of (2) we can assume that $h_{t}(1 / 2 \leqq$ $t \leqq 1)$ is made up of pieces $\left.h_{t}(1-1 / j \leqq t \leqq 1-1 /(j+1))\right)(j=2,3, \cdots)$ where $h_{t}(1-1 / j \leqq t \leqq 1-1 /(j+1)) \leqq N(\mathscr{F}, f, 1 / j) \cap \mathscr{G}$. From the proof of (1) each piece of $h_{t}$ can be chosen to have the form

$$
H_{t}^{j} h_{1-1 / j}\left(H_{t}^{j}\right)^{-1}(1-1 / j \leqq t \leqq 1-1 /(j+1))
$$

where $H_{t}^{j}(1-1 / j \leqq t \leqq 1-1 /(j+1))$ is a $1 / j$-isotopy of $\Sigma$ with $H_{1-1 / j}^{j}=$ I. Define a pseudo isotopy $H_{t}^{1}(1 / 2 \leqq t \leqq 1)$ of $\Sigma$ by the rule $H_{1 / 2}^{1}=I$, $H_{t}^{1}=H_{t}^{j} H_{1-1 / j}^{1}(1-1 / j \leqq t \leqq 1-1 /(j+1), j=2,3, \cdots)$, and

$$
H_{1}^{1}=\lim H_{t}^{1}(t \rightarrow 1) \text {. }
$$

Note that $H_{1}^{1} h_{1 / 2}\left(H_{1}^{1}\right)^{-1}=\lim h_{t}(t \rightarrow 1)=f$.

By a similar argument we obtain from the proofs of (4) and (1) an isotopy $H_{t}^{0}(0 \leqq t \leqq 1 / 2)$ of $\Sigma$ such that $H_{0}^{0}=I$ and $g_{2}=H_{1 / 2}^{0} g\left(H_{1 / 2}^{0}\right)^{-1}$. Define the pseudo isotopy $H_{t}$ by the rule $H_{t}=H_{t}^{0}(0 \leqq t \leqq 1 / 2)$ and $H_{t}=H_{t}^{1} H_{1 / 2}(1 / 2 \leqq t \leqq 1)$. For $1 / 2 \leqq t<1$ we have $H_{t} g\left(H_{t}\right)^{-1}=$ $H_{t}^{1} H_{1 / 2} g\left(H_{t}^{1} H_{1 / 2}\right)^{-1}=H_{t}^{1} g_{2}\left(H_{t}^{1}\right)^{-1}=h_{t}$; thus $f=H_{1} g H_{1}^{-1}$.

Both Bob Daverman and the referee suggested the following alternative way to obtain $H_{t}^{1}$ : Let $S$ bound crumpled cubes $C$ and $D$. Split open $\Sigma$ along $S$ and add $S \times[-1,1]$ so that $\Sigma$ is represented as the sum $C \cup S \times[-1,1] \cup D$. Define $g_{2}=f$ on $C$ and $D$, and set $g_{2}((x, t))=(x,-t)$ for $(x, t) \in S \times[-1,1]$. A result of Price's [20] provides a pseudo isotopy $H_{t}^{2}(1 / 2 \leqq t \leqq 1)$ of $\Sigma$ which shrinks the fibers of $S \times[-1,1]$ back to points and transforms $g_{2}$ to $f$ by the conjugation $f=H_{1}^{1} g_{2}\left(H_{1}^{1}\right)^{-1}$.

\section{REFERENCES}

1. J. W. Alexander, On the deformation of an n-cell, Proc. Nat. Acad. of Sci. U.S.A. 10 (1924), 6-8.

2. W. R. Alford, Uncountably many different involutions of $S^{3}$, Proc. Amer. Math. Soc. 17 (1966), 186-196.

3. R. H. Bing, A homeomorphism between the 3-sphere and the sum of two solid horned spheres, Ann. of Math. 56 (1952), 354-362.

4. L Locally tame sets are tame, Ann. of Math. 59 (1954), 145-158.

$5 . \quad$ Approximating surfaces with polyhedral ones, Ann. of Math. 65 (1957), 456-483.

6. - An alternative proof that 3-manifolds can be triangulated, Ann. of Math. 69 (1959), 37-65.

7. Conditions under which a surface in $E^{3}$ is tame, Fund. Math. 47 (1959), 105-139.

8. — Each disk in $E^{3}$ is pierced by a tame arc, Amer. J. Math. 84 (1962), 591-599.

9. Inequivalent families of periodic homeomorphisms of $E^{3}$, Ann. of Math. 80 (1964), 78-93. 
10. Improving the side approximation theorem, Trans. Amer. Math. Soc. 116 (1965), 511-525.

11. R. Craggs, Improving the intersection of polyhedra in 3-manifolds, Illinois J. Math. 12 (1968), 567-586.

12. , Building cartesian products of surfaces with [0,1] (to appear in Trans. Amer. Math. Soc.)

13. Small ambient isotopies of a 3-manifold which transform one embedding of a polyhedron into another (to appear in Fund. Math.)

14. W. Hurewicz and H. Wallman, Dimension theory, Princeton Univ. Press, 1948.

15. J. M. Kister, Isotopies in 3-manifolds, Trans. Amer. Math. Soc. 97 (1960), 213-224.

16. D. R. McMillan, Jr., Neighborhoods of surfaces in 3-manifolds, Michigan Math. J. 14 (1967), 161-170.

17. E. E. Moise, Affine structures in 3-manifolds, II, Positional properties of 2-spheres, Ann. of Math. 55 (1952), 172-176.

18. , Affine structures in 3-manifolds, V, The triangulation theorem and Hauptvermutung, Ann. of Math. 56 (1952), 96-114.

19. _ Affine structures in 3-manifolds, VIII, Invariance of the knot-type; local tame imbedding, Ann. of Math. 59 (1954), 159-170.

20. T. M. Price, Decompositions of $S^{3}$ and pseudo-isotopies, Abst. 653-201, Amer. Math. Soc. Notices 15 (1968), 136.

21. P. A. Smith, Transformations of finite period, Ann. of Math. 39 (1938), 127-164. 22. Ta-Sun Wu, On the involutions of the 3-sphere, Abst. 61T-268, Amer. Math. Soc. Notices 8 (1961), 518.

Received February 3, 1969.

THE UNIVERSITY OF ILLINOIS 



\section{PACIFIC JOURNAL OF MATHEMATICS}

\section{EDITORS}

\author{
H. SAMELSON \\ Stanford University \\ Stanford, California 94305 \\ RICHARD PIERCE \\ University of Washington \\ Seattle, Washington 98105
}

J. DugundJI

Department of Mathematics University of Southern California Los Angeles, California 90007

BASIL GORDON*

University of California

Los Angeles, California 90024

\section{ASSOCIATE EDITORS}
E. F. BECKENBACH
B. H. NEUMANN
F. WOLE
K. YOSHIDA

\section{SUPPORTING INSTITUTIONS}

\author{
UNIVERSITY OF BRITISH COLUMBIA \\ CALIFORNIA INSTITUTE OF TECHNOLOGY \\ UNIVERSITY OF CALIFORNIA \\ MONTANA STATE UNIVERSITY \\ UNIVERSITY OF NEVADA \\ NEW MEXICO STATE UNIVERSITY \\ OREGON STATE UNIVERSITY \\ UNIVERSITY OF OREGON \\ OSAKA UNIVERSITY \\ UNIVERSITY OF SOUTHERN CALIFORNIA
}

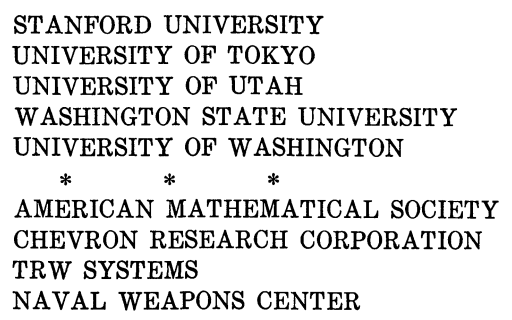

The Supporting Institutions listed above contribute to the cost of publication of this Journal, but they are not owners or publishers and have no responsibility for its content or policies.

Mathematical papers intended for publication in the Pacific Journal of Mathematics should be in typed form or offset-reproduced, (not dittoed), double spaced with large margins. Underline Greek letters in red, German in green, and script in blue. The first paragraph or two must be capable of being used separately as a synopsis of the entire paper. The editorial "we" must not be used in the synopsis, and items of the bibliography should not be cited there unless absolutely necessary, in which case they must be identified by author and Journal, rather than by item number. Manuscripts, in duplicate if possible, may be sent to any one of the four editors. Please classify according to the scheme of Math. Rev. 36, 1539-1546. All other communications to the editors should be addressed to the managing editor, Richard Arens, University of California, Los Angeles, California, 90024.

50 reprints are provided free for each article; additional copies may be obtained at cost in multiples of 50 .

The Pacific Journal of Mathematics is published monthly. Effective with Volume 16 the price per volume (3 numbers) is $\$ 8.00$; single issues, $\$ 3.00$. Special price for current issues to individual faculty members of supporting institutions and to individual members of the American Mathematical Society: $\$ 4.00$ per volume; single issues $\$ 1.50$. Back numbers are available.

Subscriptions, orders for back numbers, and changes of address should be sent to Pacific Journal of Mathematics, 103 Highland Boulevard, Berkeley, California, 94708.

PUBLISHED BY PACIFIC JOURNAL OF MATHEMATICS, A NON-PROFIT CORPORATION

Printed at Kokusai Bunken Insatsusha (International Academic Printing Co., Ltd.), 7-17, Fujimi 2-chome, Chiyoda-ku, Tokyo, Japan.

* Acting Managing Editor. 


\section{Pacific Journal of Mathematics}

\section{Vol. 32, No. $2 \quad$ February, 1970}

Harry P. Allen and Joseph Cooley Ferrar, Jordan algebras and exceptional subalgebras of the exceptional algebra $E_{6} \ldots \ldots \ldots \ldots \ldots \ldots \ldots 283$

David Wilmot Barnette and Branko Grünbaum, Preassigning the shape of a

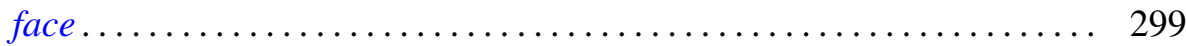

Robert Francis Craggs, Involutions of the 3-sphere which fix 2-spheres . . . . 307

David William Dean, Bor-Luh Lin and Ivan Singer, On k-shrinking and $k$-boundedly complete bases in Banach spaces ................ 323

Martin Engert, Finite dimensional translation invariant subspaces ....... 333

Kenneth Lewis Fields, On the global dimension of residue rings ......... 345

Howard Gorman, The Brandt condition and invertibility of modules ....... 351

Benjamin Rigler Halpern, A characterization of the circle and interval ..... 373

Albert Emerson Hurd, A uniqueness theorem for second order quasilinear

hyperbolic equations ............................... 415

James Frederick Hurley, Composition series in Chevalley algebras ...... 429

Meira Lavie, Disconjugacy of linear differential equations in the complex

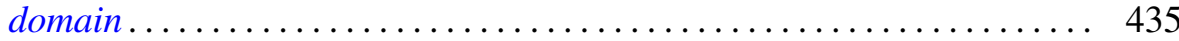

Jimmie Don Lawson, Lattices with no interval homomorphisms ......... 459

Roger McCann, A classification of center-foci ................. 467

Evelyn Rupard McMillan, On continuity conditions for functions . . . . . . . 479

Graciano de Oliveira, A conjecture and some problems on permanents .... 495

David L. Parrott and S. K. Wong, On the Higman-Sims simple group of order $44,352,000$.

Jerome L. Paul, Extending homeomorphisms ................. 517

Thomas Benny Rushing, Unknotting unions of cells .............. 521

Peter Russell, Forms of the affine line and its additive group.......... 527

Niel Shilkret, Non-Archimedean Gelfand theory ................. 541

Alfred Esperanza Tong, Diagonal submatrices of matrix maps.......... 551 